$\mathrm{e}^{+} \mathrm{e}^{-}$Collisions from Phi to Psi 2013 (PHIPSI2013)

International Journal of Modern Physics: Conference Series

Vol. 35 (2014) 1460387 (5 pages)

(C) The Authors

DOI: $10.1142 /$ S2010194514603871

\title{
HADRONIC CROSS SECTION MEASUREMENT AT CMD-3
}

\author{
R.R.AKHMETSHIN, A.V.ANISENKOV, V.M.AULCHENKO*† V.S.BANZAROV*, \\ L.M.BARKOV, N.S.BASHTOVOY* D.E.BERKAEV*; A.V.BRAGIN*, S.I.EIDELMAN*';

 \\ A.A.GREBENUK* ${ }^{* \dagger}$ D.N.GRIGORIEV ${ }^{* \dagger} ;$ E.M.GROMOV* F.V.IGNATOV* S.V.KARPOV** \\ V.F.KAZANIN*', B.I.KHAZIN*†, A.N.KIRPOTIN, I.A.KOOP*', O.A.KOVALENKO*', \\ A.N.KOZYREV, E.A.KOZYREV*; P.P.KROKOVNY*; A.E.KUZMENKO*; A.S.KUZMIN, \\ I.B.LOGASHENKO*†\$ P.A.LUKIN*† A.P.LYSENKO* K.Yu.MIKHAILOV*† V.S.OKHAPKIN*

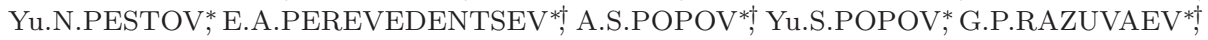 \\ Yu.A.ROGOVSKY* A.L.ROMANOV* A.A.RUBAN* N.M.RYSKULOV* A.E.RYZHENENKOV*†

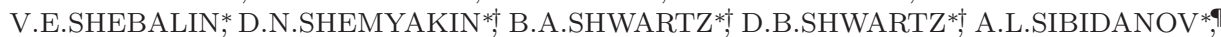 \\ P.Yu.SHATUNOV ${ }^{*}$ Yu.M.SHATUNOV ${ }^{*}$ E.P.SOLODOV ${ }^{* \dagger}$, V.M.TITOV, A.A.TALYSHEV *†, \\ A.I.VOROBIOV*, Yu.V.YUDIN, Yu.M.ZHARINOV* \\ ${ }^{*}$ Budker Institute of Nuclear Physics, SB RAS, Novosibirsk, 630090, Russia \\ $\dagger^{\dagger}$ Novosibirsk State University, Novosibirsk, 630090, Russia \\ ${ }^{\ddagger}$ Novosibirsk State Technical University, Novosibirsk, 630092, Russia \\ IUniversity of Sydney, School of Physics, Falkiner High Energy Physics, NSW 2006, Sydney, \\ Australia \\ "University of Tokyo, Department of Physics, 7-3-1 Hongo Bunkyo-ku Tokyo, 113-0033, Japan \\ $\S$ Corresponding author:I.B.Logashenko@inp.nsk.su
}

Published 18 December 2014

\begin{abstract}
The VEPP-2000 electron-positron collider was commissioned in 2010. About $60 \mathrm{pb}^{-1}$ were collected so far by CMD-3 detector in the whole available c.m. energy range from $0.32 \mathrm{GeV}$ to $2.0 \mathrm{GeV}$. The preliminary results of data analysis for various modes of $e^{+} e^{-} \rightarrow$ hadrons are discussed.
\end{abstract}

Keywords: $e^{+} e^{-}$colliders; hadrons; detectors; CMD-3.

\section{VEPP-2000 and CMD-3}

The electron-positron collider VEPP-2000 ${ }^{1-2}$ was commissioned at Budker Institute of Nuclear Physics (Novosibirsk, Russia) in 2010. The machine covers energy range from $\sqrt{s}=0.32 \mathrm{GeV}$ to $\sqrt{s}=2.0 \mathrm{GeV}$ and employs novel round beam technique to reach design luminosity up to $10^{32} \mathrm{~cm}^{-2} \mathrm{~s}^{-1}$ at $2 \mathrm{GeV}$.

Two detectors, CMD- $3^{3}$ and $\mathrm{SND},{ }^{4}$ installed in the interaction regions of the collider, started data taking by the end of 2010. Three independent data taking runs

This is an Open Access article published by World Scientific Publishing Company. It is distributed under the terms of the Creative Commons Attribution 3.0 (CC-BY) License. Further distribution of this work is permitted, provided the original work is properly cited. 
were performed so far, the whole available energy range was covered and about 60 $\mathrm{pb}^{-1}$ of integrated luminosity were collected by each detector. The physics program of experiments includes the high precision measurement of cross sections of various modes of $e^{+} e^{-} \rightarrow$ hadrons, studies of known and searchers for new vector mesons, measurement of $n \bar{n}$ and $p \bar{p}$ production cross sections near threshold and searches for exotic hadrons.

CMD-3 (Cryogenic Magnetic Detector) is a general purpose detector (Fig. 1). The tracks of charged particles are detected by the cylindrical drift chamber with a hexagonal cells. The fiducial volume for charged tracks is precisely determined by the Z-chamber, a MWPC with dual anode and cathode readout. The barrel electromagnetic calorimeter, placed outside of the superconducting solenoid $\left(0.13 \mathrm{X}_{0}\right.$, $13 \mathrm{kGs}$ ), consists of two systems: the Liquid Xenon calorimeter (about 5.4X $\mathrm{X}_{0}$ ), surrounded by the CsI crystal calorimeter (about $8.1 \mathrm{X}_{0}$ ). The LXe calorimeter has 7 layers and utilizes dual readout: the anode signals are used for measurement of the total energy deposition, while signals from the cathode strips provide information about a shower profile and are used for measurement of the coordinates of photons with high precision (about 1-2 mm). The endcap BGO crystal calorimeter (about $13.4 \mathrm{X}_{0}$ ) operates in the main magnetic field. The time-of-flight system, designed to identify slow moving particles, is placed between the two layers of barrel calorimeter. The detector is surrounded by the muon range system.

Overall, about $60 \mathrm{pb}^{-1}$ were collected so far, with about $35 \mathrm{pb}^{-1}$ above $\phi$ energy (collected in 2011-2012), about $8.5 \mathrm{pb}^{-1}$ both at $\omega$ and $\phi$ resonances, and about $9 \mathrm{pb}^{-1}$ below $\phi$ energy. Average luminosity above $10^{31} \mathrm{~cm}^{-2} \mathrm{~s}^{-1}$ was reached at VEPP-2000, which is still below the design luminosity. The main limitation at high energies is a deficit of positrons. After VEPP-2000 upgrade, started in 2013, which includes commission of the new positron injection facility and increase of the maximum energy of the booster ring, the gain in maximum luminosity up to factor of 10 is expected.

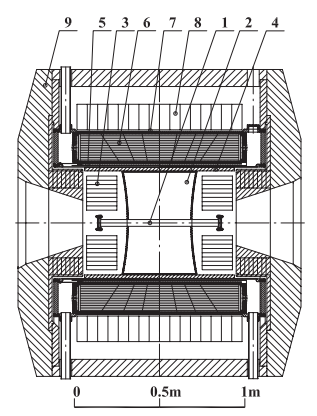

Fig. 1. CMD-3 detector: 1 - beam pipe, $2-$ drift chamber, 3 - BGO calorimeter, $4-\mathrm{Z}$ chamber, 5 - superconducting solenoid, 6 - LXe calorimeter, 7 - TOF system, 8 - CsI calorimeter, 9 - yoke. Muon range system, not shown, is placed outside the yoke.

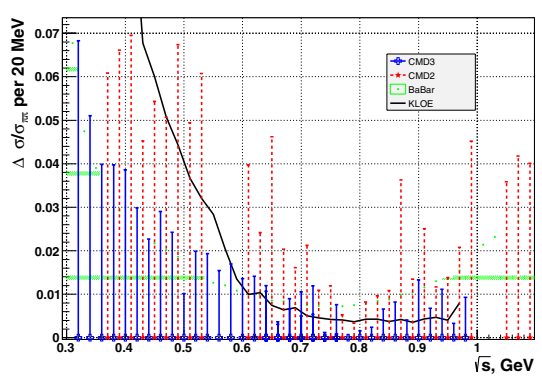

Fig. 2. Expected relative statistical precision $\sigma\left(e^{+} e^{-} \rightarrow \pi^{+} \pi^{-}\right)$from CMD-3 2013 data in comparison with previous measurements 


\section{Cross section $e^{+} e^{-} \rightarrow \pi^{+} \pi^{-}$}

The dominant mode of hadron production in $e^{+} e^{-}$collisions at energies $\sqrt{s}<1$ $\mathrm{GeV}$ is $e^{+} e^{-} \rightarrow \pi^{+} \pi^{-}$. The importance of this mode is emphasized by the fact that it is the source of the largest contribution to the uncertainty of the Standard Model evaluation of the anomalous magnetic moment of the muon. The most precise direct scan data for this channel was taken in the previous generation of experiments at Novosibirsk at VEPP-2M collider with CMD-2 and SND detectors. The high precision measurement of this mode was recently performed at KLOE and BaBar experiments using initial-state radiation (ISR) technique. The systematic precision varies between the experiments from $0.5 \%$ to $1.3 \%$. There is good overall agreement between different measurements, but there are local disagreements. The good review of the existing data is provided in the most recent calculations of the hadronic contribution to muon's $g-2 .^{5-6}$ The expected precision of the new experiment to measure $a_{\mu}$, under construction at FNAL, is $0.15 \mathrm{ppm}$, which corresponds to about $0.25 \%$ systematic uncertainty in $\sigma\left(e^{+} e^{-} \rightarrow\right.$ hadrons $)$.

The CMD-3 goal it to measure $e^{+} e^{-} \rightarrow \pi^{+} \pi^{-}$cross section with systematic uncertainty of $0.35 \%$ at $\sqrt{s}<1 \mathrm{GeV}$ and moderately larger uncertainty at higher energies. The same technique as in previous VEPP-2M measurements is used: final states with two back-to-back particles with an opposite charge are selected and then identified as $e^{+} e^{-} \rightarrow e^{+} e^{-}, \mu^{+} \mu^{-}, \pi^{+} \pi^{-}$pairs with small cosmic background. Two independent approaches are used to separate the final states: by particles momenta (at energies below $\sqrt{s}<0.7 \mathrm{GeV}$ ) and by energy deposition in the calorimeter. In order to get the cross section, number of $\pi^{+} \pi^{-}$pairs is normalized to number of $e^{+} e^{-}$pairs. The ratio of numbers of $\mu^{+} \mu^{-}$and $e^{+} e^{-}$pairs, or the measured $e^{+} e^{-} \rightarrow \mu^{+} \mu^{-}$cross-section, provides strong overall systematic test, as this ratio is well known from QED.

Several detector advantages should allow to reach the goal precision. Two independent ways of event separation provide important cross-check of systematic errors. The detector construction allows for two independent ways for precise determination of the fiducial volume, using data from Z-chamber or LXe calorimeter. Mapping of detector elements using $\gamma$ conversion and analysis of the angular distribution of $e^{+} e^{-}$pairs provide additional systematic checks of acceptance. For direct scan measurement, performed at CMD-3, it is important to know an average beam energy. A dedicated system, ${ }^{7}$ installed at VEPP-2000 at 2012, monitors beam energy in real time with $50 \mathrm{keV}$ precision using Compton backscattering of the laser photons. In order to test the calculation of the radiative corrections, ${ }^{8}$ it is important to compare different approaches and software packages and to perform a detailed comparison of the radiative tails to the data.

In the first half of 2013 a large sample of data dedicated to high precision measurement of $\sigma\left(e^{+} e^{-} \rightarrow \pi^{+} \pi^{-}\right)$were collected at $\sqrt{s}<1 \mathrm{GeV}$. The integrated statistics is few times of that at CMD-2 and is at the level of statistics collected at BaBar and KLOE (Fig. 2). The data analysis is in progress. 


\section{Other modes}

At energies above $\phi$-meson dipion production is not the dominant mode of $e^{+} e^{-}$ annihilation to hadrons. The majority of the modes have several pions (and kaons) in the final state, have rich dynamics with many intermediate states. For most of the cases, the precision of the cross section measurement is limited by model dependence. In 2011 and 2012 about $35 \mathrm{pb}^{-1}$ were collected at $\sqrt{s}>1 \mathrm{GeV}$, and there are plans to collect factor of 10 or more data after VEPP-2000 upgrade. High statistics will allow to study dynamics of the complicated multi-hadron final states, which will reduce model uncertainty.

The total cross section of the process $e^{+} e^{-} \rightarrow 3\left(\pi^{+} \pi^{-}\right)$has been measured using $22 \mathrm{pb}^{-1}$ of integrated luminosity collected with the CMD-3 detector in the $\sqrt{s}=1.5$ 2.0 GeV energy range. ${ }^{9}$ The measured cross section is shown in Fig. 3 where a sharp dip is seen near $p \bar{p}$ threshold. It was found that the observed production mechanism can be described by the production of one $\rho(770)$ with four remaining pions in the S-wave distributed according to phase space. It was also observed that the production dynamics change in the $\sqrt{s}=1.7 \div 1.9 \mathrm{GeV}$ energy range, which demands a further study. Detailed analysis of the production dynamics will be performed in the combined analysis of the processes $e^{+} e^{-} \rightarrow 3\left(\pi^{+} \pi^{-}\right)$and $e^{+} e^{-} \rightarrow 2\left(\pi^{+} \pi^{-} \pi^{0}\right)$. The preliminary results on $2\left(\pi^{+} \pi^{-} \pi^{0}\right)$ are shown in Fig. 4.

A large sample of $e^{+} e^{-} \rightarrow 2\left(\pi^{+} \pi^{-}\right)$events is collected. A production mechanism through $a_{1}(1260) \pi$ is confirmed, while small admixtures of $\rho(770) f_{0}(600)$, $\rho(770) f_{0}(980)$ and $a_{2}(1320) \pi$ are seen. There is clear signal of $\omega \pi^{0}$ events in the collected $e^{+} e^{-} \rightarrow \pi^{+} \pi^{-} \pi^{0} \pi^{0}$ sample. Non- $\omega \pi^{0}$ events are dominated by $a_{1}(1260) \pi$ with additional small contributions from $\rho^{+} \rho^{-}$and $\rho(770) f_{0}(980)$. In order to measure $\sigma\left(e^{+} e^{-} \rightarrow 4 \pi\right)$, all these production mechanisms should be taken into account in simulation.

Above $\phi$ energy there are many modes of $e^{+} e^{-} \rightarrow$ hadrons with kaons in the final state. Preliminary results of CMD-3 analysis of $e^{+} e^{-} \rightarrow K^{+} K^{-} \pi^{+} \pi^{-}$ cross section are presented in separate contribution in this proceedings. ${ }^{10}$ This

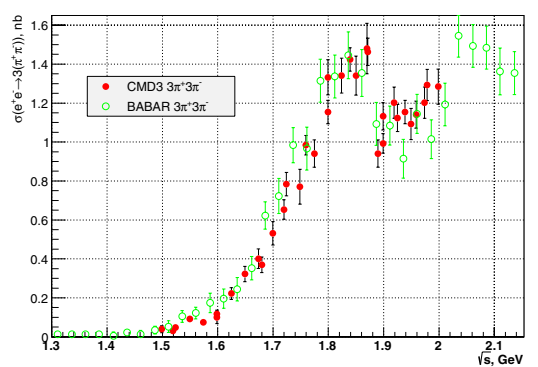

Fig. 3. Measurement of the $e^{+} e^{-} \rightarrow 3\left(\pi^{+} \pi^{-}\right)$ cross section



Fig. 4. Preliminary results of measurement of the $e^{+} e^{-} \rightarrow 2\left(\pi^{+} \pi^{-} \pi^{0}\right)$ cross section. Dots CMD-3 data, circles - BaBar data. 
mode is especially interesting because many intermediate states contribute to it: $K^{*}(1270) K \rightarrow K^{*}(892) K \pi, K^{*}(1400) K \rightarrow K^{*}(892) K \pi, K^{*}(1270) K \rightarrow \rho K K$, $K^{*}(892) K^{*}(892), \phi \pi \pi$.

The maximum energy of VEPP-2000 is above $p \bar{p}$ and $n \bar{n}$ masses, which allows for direct measurement of nucleon formfactors near threshold. Currently, about $6 \mathrm{pb}^{-1}$ were collected for $\sqrt{s} \geq 2 m_{p}$. The preliminary results of the $p \bar{p}$ production cross section measurement are presented in separate contribution in this proceedings. ${ }^{11}$ CMD-3 plans to collect two orders of magnitude more data in this energy range, which will allow to measure $\left|G_{E} / G_{M}\right|$ ratio with $5-10 \%$ statistical precision.

\section{Conclusion}

The CMD-3 detector has collected data in the whole energy range available at VEPP-2000. Data analysis is in progress and first results are already published $(6 \pi)$. The improved CMD-3 detector performance, compared to its predecessor CMD-2, should allow to obtain small systematic error, important for $a_{\mu}$ evaluation. Factor of 10 improvement in luminosity is expected after new positron injection complex is commissioned.

\section{Acknowledgments}

This work is supported in part by the RFBR grants 11-02-00112-a, 11-02-00328-a, 12-02-00065-a, 12-02-01032-a, 12-02-01250-a. 12-02-31498-mol-a, 12-02-31499-mola, 12-02-31501-mol-a, 13-02-00215-a, and the DFG grant GZ: HA 1457/7-2.

\section{References}

1. Yu. M. Shatunov et al., Conf. Proc. C 0006262 (2000) 439.

2. D. Berkaev et al., Nucl. Phys. Proc. Suppl. 225-227 (2012) 303.

3. B. I. Khazin et al., Nucl. Phys. Proc. Suppl. 181-182 (2008) 376.

4. M. N. Achasov et al., Nucl. Instrum. Meth. A 598 (2009) 31.

5. K. Hagiwara, R. Liao, A. D. Martin, D. Nomura, T. Teubner, J. Phys. G 38 (2011) 085003.

6. M. Davier, A. Hoecker, B. Malaescu, Z. Zhang1, Eur. Phys. J C 71 (2011) 1515, erratum Eur. Phys. J. C 72 (2012) 1874.

7. E. V. Abakumova et al., Phys. Rev. Lett. 110 (2013) 140402

8. S. Actis et al., Eur. Phys. J. C 66 (2010) 585

9. R. R. Akhmetshin et al., Phys. Lett. B 723 (2013) 82

10. R. R. Akhmetshin et al., Study of the process $e^{+} e^{-} \rightarrow K^{+} K^{-} \pi^{+} \pi^{-}$with the CMD-3 detector at the VEPP-2000, to appear in these proceedings.

11. R. R. Akhmetshin et al.,Measurement of the $e^{+} e^{-} \rightarrow p \bar{p}$ cross section with CMD-3 detector at VEPP-2000, to appear in these proceedings. 